\title{
CORRECTION
}

\section{Correction to: Potentially modifiable factors contributing to outcome from acute respiratory distress syndrome: the LUNG SAFE study}

\author{
John G. Laffey ${ }^{1,2^{*}}$ (D) Giacomo Bellani ${ }^{3,4}$, Tài Pham ${ }^{5,6,7}$, Eddy Fan ${ }^{8,9}$, Fabiana Madotto ${ }^{10}$, Ednan K. Bajwa ${ }^{11}$, \\ Laurent Brochard ${ }^{12,13}$, Kevin Clarkson ${ }^{14}$, Andres Esteban ${ }^{15}$, Luciano Gattinoni ${ }^{16}$, Frank van Haren ${ }^{17}$, \\ Leo M. Heunks ${ }^{18}$, Kiyoyasu Kurahashi ${ }^{19}$, Jon Henrik Laake ${ }^{20}$, Anders Larsson ${ }^{21}$, Daniel F. McAuley ${ }^{22}$, \\ Lia McNamee ${ }^{22}$, Nicolas Nin ${ }^{15}$, Haibo Qiu ${ }^{23}$, Marco Ranieri ${ }^{24}$, Gordon D. Rubenfeld ${ }^{25}$, B. Taylor Thompson ${ }^{11}$, \\ Hermann Wrigge ${ }^{26}$, Arthur S. Slutsky ${ }^{12,13,27}$, Antonio Pesenti ${ }^{28,29}$, The LUNG SAFE Investigatorsthe ESICM Trials \\ Group
}

๑) 2017 Springer-Verlag GmbH Germany and ESICM

\section{Correction to: Intensive Care Med (2016) 42:1865-1876 DOI 10.1007/s00134-016-4571-5}

The members of the LUNG SAFE Investigators and the ESICM Trials Group were provided in such a way that they could not be indexed as collaborators on PubMed. The publisher apologizes for this error and is pleased to list the members of the groups here:

\section{Appendix 1: NATIONAL COORDINATORS:}

Argentina: Fernando Rios; Australia/New Zealand: Frank Van Haren; Belgium: T. Sottiaux, P. Depuydt; Bolivia: Fredy S. Lora; Brazil: Luciano Cesar Azevedo; Canada: Eddy Fan; Chile: Guillermo Bugedo; China: Haibo Qiu; Colombia: Marcos Gonzalez; Costa Rica: Juan Silesky; Czech Republic: Vladimir Cerny; Denmark: Jonas Nielsen; Ecuador: Manuel Jibaja; France: Tài Pham; Germany: Hermann Wrigge; Greece: Dimitrios Matamis; Guatemala: Jorge Luis Ranero; India:

\footnotetext{
*Correspondence: laffeyj@smh.ca

${ }^{1}$ Departments of Anesthesia and Critical Care Medicine, Keenan Research Centre for Biomedical Science, St Michael's Hospital, Toronto, Canada Full author information is available at the end of the article
}

The original article can be found online at https://doi.org/10.1007/ s00134-016-4571-5.
Pravin Amin; Iran: S. M. Hashemian; Ireland: Kevin Clarkson; Italy: Giacomo Bellani; Japan: Kiyoyasu Kurahashi; Mexico: Asisclo Villagomez; Morocco: Amine Ali Zeggwagh; Netherlands: Leo M. Heunks; Norway: Jon Henrik Laake; Philippines: Jose Emmanuel Palo; Portugal: Antero do Vale Fernandes; Romania: Dorel Sandesc; Saudi Arabia: Yaasen Arabi; Serbia: Vesna Bumbasierevic; Spain: Nicolas Nin, Jose A. Lorente; Sweden: Anders Larsson; Switzerland: Lise Piquilloud; Tunisia: Fekri Abroug; United Kingdom: Daniel F. McAuley, Lia McNamee; Uruguay: Javier Hurtado; USA: Ed. Bajwa; Venezuela: Gabriel Démpaire.

\section{NATIONAL SOCIETIES/NETWORKS ENDORSING THE STUDY:}

ANZICS Clinical Trials Group, Réseau Européen de Recherche en Ventilation Artificielle (ReVA Network); Irish Critical Care Trials Group; Société de Réanimation de Langue Française (SRLF); Société Française d'Anesthésie et de Réanimation (SFAR); Società Italiana Anestesia, Analgesia, Rianimazione e Terapia Intensiva (SIAARTI); The Japanese Society of Intensive Care Medicine (JSICM); Nonprofit Organization Japanese Society 
of Education for Physicians and Trainees in Intensive Care (JSEPTIC); UK Intensive Care Society.

\section{Appendix 2: List of LUNG SAFE Investigators}

STUDY COORDINATION: Guy M. Francois (European Society of Intensive Care Medicine, Brussels, Belgium).

DATA REVISION AND MANAGEMENT: Francesca Rabboni (University of Milan-Bicocca, Monza, Italy), Fabiana Madotto (University of Milan-Bicocca, Monza, Italy), Sara Conti (University of Milan-Bicocca, Monza, Italy).

\section{SITE INVESTIGATORS BY COUNTRY:}

ALBANIA: Uhc Mother Theresa (Tirana): Hektor Sula, Lordian Nunci; University Hospital Shefqet Ndroqi (Tirana): Alma Cani;

ARGENTINA: Clinica De Especialidades (Villa Maria): Alan Zazu; Hospital Dr Julio C. Perrando (Resistencia): Christian Dellera, Carolina S. Insaurralde; Sanatorio Las Lomas (San Isidro, Buenos Aires): Risso V. Alejandro; Sanatorio De La Trinidad San Isidro (San Isidro): Julio Daldin, Mauricio Vinzio; Hospital Español De Mendoza (Godoy Cruz, Mendoza): Ruben O. Fernandez; Hospital Del Centenario (Rosario): Luis P. Cardonnet, Lisandro R. Bettini; San Antonio (Gualeguay (Entre Rios)): Mariano Carboni Bisso, Emilio M. Osman; Cemic (Buenos Aires): Mariano G. Setten, Pablo Lovazzano; Hospital Universitrario Austral (Pilar): Javier Alvarez, Veronica Villar; Hospital Por + Salud (Pami) Dr. Cesar Milstein (Buenos Aires): Norberto C. Pozo, Nicolas Grubissich; Sanatorio Anchorena (Buenos Aires): Gustavo A. Plotnikow, Daniela N. Vasquez; Sanatorio De La Trinidad Mitre (Buenos Aires): Santiago Ilutovich, Norberto Tiribelli; Hospital Luis Lagomaggiore (Mendoza): Ariel Chena, Carlos A. Pellegrini; H.I.G.A San Martín (La Plata): María G. Saenz, Elisa Estenssoro; Hospital Misericordia (Cordoba): Matias Brizuela, Hernan Gianinetto; Sanatorio Juncal (Temperley): Pablo E. Gomez, Valeria I. Cerrato; Hospital D. F. Santojanni (Buenos Aires): Marco G. Bezzi, Silvina A. Borello; Hospital Alejandro Posadas (Buenos Aires): Flavia A. Loiacono, Adriana M. Fernandez.

AUSTRALIA: St. Vincent's Hospital, Sydney (Darlinghurst): Serena Knowles, Claire Reynolds; St George Public Hospital (Kogarah): Deborah M. Inskip, Jennene J. Miller; Westmead Hospital (Westmead): Jing Kong, Christina Whitehead; Flinders Medical Centre (Bedford Park, South Australia): Shailesh Bihari; John Hunter Hospital (Newcastle): Aylin Seven, Amanda Krstevski; Canberra Hospital (Garran): Helen J. Rodgers, Rebecca
T. Millar; Calvary Mater Newcastle (Waratah): Toni E. Mckenna, Irene M. Bailey; Cabrini Hospital (Melbourne): Gabrielle C. Hanlon; Liverpool Hospital (Liverpool): Anders Aneman, Joan M. Lynch; Coffs Harbour Health Campus (Coffs Harbour): Raman Azad, John Neal; Sir Charles Gairdner Hospital (Nedlands): Paul W. Woods, Brigit L. Roberts; Concord Hospital (Concord): Mark R. Kol, Helen S. Wong.

AUSTRIA: General Hospital of Vienna/Medical University of Vienna (Vienna): Katharina C. Riss, Thomas Staudinger.

BELGIUM: Cliniques universitaires St Luc, UCL (Brussels): Xavier Wittebole, Caroline Berghe; CHU DinantGodinne (Yvoir): Pierre A. Bulpa, Alain M. Dive; AZ Sint Augustinus Veurne (Veurne): Rik Verstraete, Herve Lebbinck; Ghent University Hospital (Ghent): Pieter Depuydt, Joris Vermassen; University Hospitals Leuven (Leuven): Philippe Meersseman, Helga Ceunen.

BRAZIL: Hospital Renascentista (Pouso Alegre): Jonas I. Rosa, Daniel O. Beraldo; Vitoria Apart Hospital (Serra): Claudio Piras, Adenilton M. Rampinelli; Hospital Das Clinicas (São Paulo): Antonio P. Nassar Jr.; Hospital Geral Do Grajaù (São Paulo): Sergio Mataloun, Marcelo Moock; Evangelical Hospital (Cachoeiro De Itapemirim/ Espírito Santo): Marlus M. Thompson, Claudio H. Gonçalves; Hospital Moinhos De Vento (Porto Alegre): Ana Carolina P. Antônio, Aline Ascoli; Hospital Alvorada Taguatinga (Taguatinga): Rodrigo S. Biondi, Danielle C. Fontenele; Complexo Hospitalar Mngabeira Tarcisio Burity (Joao Pessoa): Danielle Nobrega, Vanessa M. Sales.

BRUNEI DARUSSALAM: Raja Isteri Pengiran Anak Saleha (RIPAS) Hospital (Bandar Seri Begawan): Dr Suresh Shindhe, Dr Dk Maizatul Aiman, B. Pg Hj Ismail.

CANADA: Medical-Surgical ICU of St Michael's Hospital (Toronto): John Laffey, Francois Beloncle; St. Josephs Health Centre (Toronto): Kyle G. Davies, Rob Cirone; Sunnybrook Health Sciences Center (Toronto): Venika Manoharan, Mehvish Ismail; Toronto Western Hospital (Toronto): Ewan C. Goligher, Mandeep Jassal; Medical Surgical ICU of the Toronto General Hospital (Toronto): Erin Nishikawa, Areej Javeed; Cardiovascular ICU of St Michael's Hospital (Toronto): Gerard Curley, Nuttapol Rittayamai; Cardiovascular ICU of the Toronto General Hospital (Toronto): Matteo Parotto, Niall D. Ferguson; Mount Sinai Hospital (Toronto): Sangeeta Mehta, Jenny Knoll; Trauma-Neuro ICU of St Michael's Hospital (Toronto): Antoine Pronovost, Sergio Canestrini. 
CHILE: Hospital Clínico Pontificia Universidad Católica De Chile (Santiago): Alejandro R. Bruhn, Patricio H. Garcia; Hospital Militar De Santiago (Santiago): Felipe A. Aliaga, Pamela A. Farías; Clinica Davila (Santiago): Jacob S. Yumha; Hospital Guillermo Grant Benavente (Concepcion): Claudia A. Ortiz, Javier E. Salas; Clinica Las Lilas (Santiago): Alejandro A. Saez, Luis D. Vega; Hospital Naval Almirante Nef (Viña Del Mar): Eduardo F. Labarca, Felipe T. Martinez; Hospital Luis Tisné Brousse (Penanolen): Nicolás G. Carreño, Pilar Lora.

CHINA: The Second Affiliated Hospital of Harbin Medical University (Harbin): Haitao Liu; Nanjing Zhong-Da Hospital, Southeast University (Nanjing): Haibo Qiu, Ling Liu; The First Affiliated Hospital of Anhui Medical University (Hefei): Rui Tang, Xiaoming Luo; Peking University People's Hospital (Beijing): Youzhong An, Huiying Zhao; Fourth Affiliated Hospital of Harbin Medical University (Harbin): Yan Gao, Zhe Zhai; Nanjing Jiangbei Peoples Hospital Affiliated to Medical School of Southeast University (Nanjing): Zheng L. Ye, Wei Wang; The First Affiliated Hospital of Dalian Medical University (Dalian): Wenwen Li, Qingdong Li; Subei Peoples Hospital of Jiangsu Province (Yangzhou): Ruiqiang Zheng; Jinling Hospital (Nanjing): Wenkui Yu, Juanhong Shen; Urumqi General Hospital (Urumqi): Xinyu Li; Intensive Care Unit, First Affiliated Hospital of Wanna Medical College, Yijishan Hospital, (Wuhu): Tao Yu, Weihua Lu; Sichuan Provincial Peoples Hospital (Chengdu): Ya Q. Wu, Xiao B. Huang; Hainan Province Peoples Hospital (Haikou): Zhenyang He; Peoples Hospital of Jiangxi Province (Nanchang): Yuanhua Lu; Qilu Hospital of Shandong University (Jinan): Hui Han, Fan Zhang; Zhejiang Provincial Peoples Hospital (Hangzhou): Renhua Sun; The First Affiliated Hospital of Bengbu Medical College (Bengbu, Anhui): Hua X. Wang, Shu H. Qin; Nanjing Municipal Government Hospital (Nanjing): Bao H. Zhu, Jun Zhao; The First Hospital of Lanzhou University (Lanzhou): Jian Liu, Bin Li; The First Affiliated Hospital of Chongqing University of Medical Science (Chongqing): Jing L. Liu, Fa C. Zhou; Xuzhou Central Hospital, Jiangsu Province, China (Xuzhou): Qiong J. Li, Xing Y. Zhang; The First Peoples Hospital of Foshan (Foshan): Zhou Li-Xin, Qiang Xin-Hua; The First Affiliated Hospital of Guangxi Medical University (Nanning): Liangyan Jiang; Renji Hospital, Shanghai Jiao Tong University School of Medicine (Shanghai): Yuan N. Gao, Xian Y. Zhao; First Hospital of Shanxi Medical University (Taiyuan): Yuan Y. Li, Xiao L. Li; Shandong Provincial Hospital (Jinan): Chunting Wang, Qingchun Yao; Fujian Provincial Hospital (Fuzhou): Rongguo Yu, Kai Chen; Henan Provincial People's Hospital (Zhengzhou): Huanzhang Shao, Bingyu Qin; The Second Affiliated Hospital of Kunming Medical
University (Kunming City): Qing Q. Huang, Wei H. Zhu; Xiangya Hospital, Central South University (Changsha): Ai Y. Hang, Ma X. Hua; The First Affiliated Hospital of Guangzhou Medical University (Guangzhou): Yimin Li, Yonghao Xu; Peoples Hospital of Hebei Province (Shijiazhuang): Yu D. Di, Long L. Ling; Guangdong General Hospital (Guangzhou): Tie H. Qin, Shou H. Wang; Beijing Tongren Hospital (Beijing): Junping Qin; Jiangsu Province Hospital (Nanjing): Yi Han, Suming Zhou.

COLOMBIA: Fundación Valle Del Lili (Cali): Monica P. Vargas.

COSTA RICA: Hospital San Juan De Dios: Juan I. Silesky Jimenez, Manuel A. González Rojas; Hospital San Juan De Dios (San José): Jaime E. Solis-Quesada, Christian M. Ramirez-Alfaro.

CZECH REPUBLIC: University Hospital of Ostrava (Ostrava): Jan Máca, Peter Sklienka.

DENMARK: Aarhus Universitetshospital (Aarhus N): Jakob Gjedsted, Aage Christiansen; Rigshospitalet: Jonas Nielsen.

ECUADOR: Hospital Militar (Quito): Boris G. Villamagua, Miguel Llano.

FRANCE: Clinique du Millenaire (Montpellier): Philippe Burtin, Gautier Buzancais; Centre Hospitalier (Roanne): Pascal Beuret, Nicolas Pelletier; CHU d'Angers (Angers): Satar Mortaza, Alain Mercat; Hôpital Marc Jacquet (Melun): Jonathan Chelly, Sébastien Jochmans; CHU Caen (Caen): Nicolas Terzi, Cédric Daubin; Henri Mondor Hospital (Créteil): Guillaume Carteaux, Nicolas de Prost; Cochin Hospital (Paris): Jean-Daniel Chiche, Fabrice Daviaud; Hôpital Tenon (Paris): Tai Pham, Muriel Fartoukh; CH Mulhouse-Emile Muller (Mulhouse): Guillaume Barberet, Jerome Biehler; Archet 1 University Hospital (Nice): Jean Dellamonica, Denis Doyen; Hospital Sainte Musse (Toulon): Jean-Michel Arnal, Anais Briquet; Hopital Nord-Réanimation des Détresses Respiratoires et Infections Sévères (Marseille): Sami Hraiech, Laurent Papazian; HEGP (Paris): Arnaud Follin; Louis Mourier Hospital (Colombes): Damien Roux, Jonathan Messika; Centre Hospitalier de Dax (Dax): Evangelos Kalaitzis; Réanimation Médicale, GH Pitié-Salpêtrière (Paris): Laurence Dangers, Alain Combes; Ap-Hp Ambroise Paré (Boulogne-Billancourt): Siu-Ming Au; University Hospital Rouen (Rouen): Gaetan Béduneau, Dorothée Carpentier; CHU Amiens (Amiens-Salouel): Elie H. Zogheib, Herve Dupont; Centre Hospitalier Intercommunal Robert Ballanger (Aulnay Sous Bois): 
Sylvie Ricome, Francesco L. Santoli; Centre Hospitalier René Dubos (Pontoise): Sebastien L. Besset; CHI Portes de l'Oise (Beaumont Sur Oise): Philippe Michel, Bruno Gelée; Archet 2 University Hospital (Nice): Pierre-Eric Danin, Bernard Goubaux; Centre Hospitalier Pierre Oudot (Bourgoin Jallieu): Philippe J. Crova, Nga T. Phan; $\mathrm{CH}$ Dunkerque (Dunkerque): Frantz Berkelmans; Centre Hospitalier de Belfort Montbéliard (Belfort): Julio C. Badie, Romain Tapponnier; Centre Hospitalier Emile Muller (Mulhouse): Josette Gally, Samy Khebbeb; Hôpital de Hautepierre-Hôpitaux Universitaires de Strasbourg (Strasbourg): Jean-Etienne Herbrecht, Francis Schneider; Centre Hospitalier de Dieppe (Dieppe): Pierre-Louis M. Declercq, Jean-Philippe Rigaud; Bicetre (Le KremlinBicetre): Jacques Duranteau, Anatole Harrois; CHU Gabriel Montpied (Clermont-Ferrand): Russell Chabanne, Julien Marin; CHU Estaing (Clermont-Ferrand): Charlene Bigot, Sandrine Thibault; CHI Eure-Seine Evreux (Evreux): Mohammed Ghazi, Messabi Boukhazna; Centre Hospitalier d Châlons en Champagne (Châlons en Champagne): Salem Ould Zein; CH Beauvais (Beauvais): Jack R. Richecoeur, Daniele M. Combaux; Centre Hospitalier Le Mans (Le Mans): Fabien Grelon, Charlene Le Moal; Hôpital Fleyriat (Bourg en Bresse): Elise P. Sauvadet, Adrien Robine; Hôpital Saint Louis (Paris): Virginie Lemiale, Danielle Reuter; Service de Pneumologie Pitié-Salpétrière (Paris): Martin Dres, Alexandre Demoule; Centre Hospitalier Gonesse (Gonesse): Dany Goldgran-Toledano; Hôpital Croix Rousse (Lyon): Loredana Baboi, Claude Guérin.

GERMANY: St. Nikolaus-Stiftshospital (Andernach): Ralph Lohner; Fachkrankenhaus Coswig $\mathrm{GmbH}$ (Coswig):Jens Kraßler, Susanne Schäfer; University Hospital Frankfurt (Frankfurt am Main): Kai D. Zacharowski, Patrick Meybohm; Department of Anaesthesia and Intensive Care Medicine, University Hospital of Leipzig (Leipzig): Andreas W. Reske, Philipp Simon; Asklepios Klinik Langen (Langen): Hans-Bernd F. Hopf, Michael Schuetz; Städtisches Krankenhaus Heinsberg (Heinsberg): Thomas Baltus.

GREECE: Hippokration General Hospital of Athens (Athens): Metaxia N. Papanikolaou, Theonymfi G. Papavasilopoulou; Gh Ahepa (Thessaloniki): Giannis A. Zacharas, Vasilis Ourailogloy; Hippokration General Hospital of Thessaloniki (Thessaloniki): Eleni K. Mouloudi, Eleni V. Massa; Hospital General of Kavala (Kavala): Eva O. Nagy, Electra E. Stamou; Papageorgiou General Hospital (Thessaloniki): Ellada V. Kiourtzieva, Marina A. Oikonomou.
GUATEMALA: Hospital General De Enfermedades, Instituto Guatemalteco De Seguridad Social (Ciudad De Guatemala): Luis E. Avila; Centro Médico Militar (Guatemala): Cesar A. Cortez, Johanna E. Citalán.

INDIA: Deenanath Mangeshkar Hospital and Research Center (Pune): Sameer A. Jog, Safal D. Sable; Care Institute of Medical Sciences (CIMS) Hospital (Ahmedabad): Bhagyesh Shah; Sanjay Gandhi Postgraduate Institute of Medical Sciences (SGPGIMS) (Lucknow): Mohan Gurjar, Arvind K. Baronia; Rajasthan Hospital (Ahmedabad): Mohammedfaruk Memon; National Institute of Mental Health and Neuro Sciences (NIMHANS) (Bangalore): Radhakrishnan Muthuchellappan, Venkatapura J. Ramesh; Anaesthesiology Unit of the Kasturba Medical College and Department of Respiratory Therapy, SHOAS, Manipal University (Manipal): Anitha Shenoy, Ramesh Unnikrishnan; Sanjeevan Hospital (Pune): Subhal B. Dixit, Rachana V. Rhayakar; Apollo Hospitals (Chennai): Nagarajan Ramakrishnan, Vallish K. Bhardwaj; Medicine Unit of the Kasturba Medical College and Department of Respiratory Therapy, SHOAS, Manipal University (Manipal): Heera L. Mahto, Sudha V. Sagar; G Kuppuswamy Naidu Memorial Hospital (Coimbatore): Vijayanand Palaniswamy, Deeban Ganesan.

IRAN: NRITLD/Masih Daneshvari (Tehran): Seyed Mohammadreza Hashemian, Hamidreza Jamaati; Milad Hospital (Tehran): Farshad Heidari.

IRELAND: St Vincent's University Hospital (Dublin): Edel A. Meaney, Alistair Nichol; Mercy University Hospital (Cork): Karl M. Knapman, Donall O'Croinin; Cork University Hospital (Cork): Eimhin S. Dunne, Dorothy M. Breen; Galway University Hospital (Galway): Kevin P. Clarkson, Rola F. Jaafar; Beaumont Hospital (Dublin): Rory Dwyer, Fahd Amir; Mater Misericordiae University Hospital (Dublin): Olaitan O. Ajetunmobi, Aogan C. O'Muircheartaigh; Tallaght Hospital (Dublin): Colin S. Black, Nuala Treanor; Saint James's Hospital (Dublin): Daniel V. Collins, Wahid Altaf.

ITALY: Santa Maria delle Croci Hospital (Ravenna): Gianluca Zani, Maurizio Fusari; Arcispedale Sant'Anna Ferrara (Ferrara): Savino Spadaro, Carlo A. Volta; Ospedale Profili (Fabriano) (An): Romano Graziani, Barbara Brunettini; Umberto I Nocera Inferiore (Nocera Inferiore Salerno): Salvatore Palmese; Azienda Ospedaliera San Paolo-Polo Universitario-Università degli Studi di Milano (Milan): Paolo Formenti, Michele Umbrello; Sant'Anna (San Fermo Della Battaglia (Co)): 
Andrea Lombardo; Spedali Civili Brescia (Brescia): Elisabetta Pecci, Marco Botteri; Fondazione IRCCS Ca Granda, Ospedale Maggiore Policlinico (Milan): Monica Savioli, Alessandro Protti; University Campus Bio-Medico of Rome (Rome): Alessia Mattei, Lorenzo Schiavoni; Azienda Ospedaliera "Mellino Mellini" (Chiari (BS)): Andrea Tinnirello, Manuel Todeschini; Policlinico P. Giaccone, University of Palermo (Palermo): Antonino Giarratano, Andrea Cortegiani; Niguarda Cà Granda Hospital (Milan): Sara Sher, Anna Rossi; A.Gemelli University Hospital (Rome): Massimo M. Antonelli, Luca M. Montini; Ospedale "Sandro Pertini" (Rome): Paolo Casalena, Sergio Scafetti; ISMeTT IRCCS UPMC (Palermo): Giovanna Panarello, Giovanna Occhipinti; Ospedale San Gerardo (Monza): Nicolò Patroniti, Matteo Pozzi; Santa Maria Della Scaletta (Imola): Roberto R. Biscione, Michela M. Poli; Humanitas Research Hospital (Rozzano): Ferdinando Raimondi, Daniela Albiero; Ospedale Desio-Ao Desio-Vimercate (Desio): Giulia Crapelli, Eduardo Beck; Pinetagrande Private Hospital (Castelvolturno): Vincenzo Pota, Vincenzo Schiavone; Irccs San Martino Ist (Genova): Alexandre Molin, Fabio Tarantino; Ospedale San Raffaele (Milano): Giacomo Monti, Elena Frati; Ospedali Riuniti Di Foggia (Foggia): Lucia Mirabella, Gilda Cinnella; Azienda Ospedaliera Luigi Sacco-Polo Universitario (Milano): Tommaso Fossali, Riccardo Colombo; A.O.U. Città della Salute e della Scienza di Torino (Turin): Pierpaolo Terragni Ilaria Pattarino; Università degli Studi di Pavia-Fondazione IRCCS Policlinico San Matteo (Pavia): Francesco Mojoli, Antonio Braschi; Ao Ospedale Civile Legnano (Legnano): Erika E. Borotto; Arnas Ospedale Civico Di Cristina Benfratelli (Palermo): Andrea N. Cracchiolo, Daniela M. Palma; Azienda Ospedaliera Della Provincia Di Lecco-Ospedale “A. Manzoni” (Lecco): Francesco Raponi, Giuseppe Foti; A.O. Provincia Di Lecco-Ospedale Alessandro Manzoni (Lecco): Ettore R. Vascotto, Andrea Coppadoro; Cliniche Universitarie Sassari (Sassari): Luca Brazzi, Leda Floris; IRCCS Policlinico San Matteo (Pavia): Giorgio A. Iotti, Aaron Venti.

JAPAN: Yokohama City University Hospital (Yokohama): Osamu Yamaguchi, Shunsuke Takagi; Toyooka Hospital (Toyooka City, Hyogo Prefecture): Hiroki N. Maeyama; Chiba University Hospital (Chiba City): Eizo Watanabe, Yoshihiro Yamaji; Okayma University Hospital (Okayama): Kazuyoshi Shimizu, Kyoko Shiozaki; Japanese Foundation for Cancer Research, Cancer Institute Hospital, Department of Emergency Medicine and Critical Care (Tokyo): Satoru Futami; Ibaraki Prefectural Central Hospital (Kasama): Sekine Ryosuke; Tohoku University Hospital (Sendai-Shi): Koji Saito, Yoshinobu Kameyama; Tokyo Medical University Hachioji Medical Center
(Hachioji, Tokyo): Keiko Ueno; Tokushima University Hospital (Tokushima): Masayo Izawa, Nao Okuda; Maebashi Red Cross Hospital (Gunma Maebashi): Hiroyuki Suzuki, Tomofumi Harasawa; Urasoe General Hospital (Urasoe): Michitaka Nasu, Tadaaki Takada; Ohta General Hospital Foundation Ohta Nishinouchi Hospital (Fukushima): Fumihito Ito; Jichi Medical University Hospital (Shimotsuke): Shin Nunomiya, Kansuke Koyama; Mito Kyodo General Hospital, Tsukuba University Hospital Mito Medical Center (Mito): Toshikazu Abe; Sendai City Hospital (Sendai): Kohkichi Andoh, Kohei Kusumoto; Ja Hiroshima General Hospital (Hatsukaichi City, Hiroshima): Akira Hirata, Akihiro Takaba; Yokohama Rosai Hospital (Yokohama): Hiroyasu Kimura; Nagasaki University Hospital (Nagasaki): Shuhei Matsumoto, Ushio Higashijima; Niigata University Medical and Dental Hospital (Niigata): Hiroyuki Honda, Nobumasa Aoki; Mie University Hospital (Tsu, Mie): Hiroshi Imai; Yamaguchi University Hospital (Ube, Yamaguchi): Yasuaki Ogino, Ichiko Mizuguchi; Saiseikai Kumamoto Hospital (Kumamoto City): Kazuya Ichikado; Shinshu University School of Medicine (Matsumoto City): Kenichi Nitta, Katsunori Mochizuki; Kuki General Hospital (Kuki): Tomoaki Hashida; Kyoto Medical Center (Kyoto): Hiroyuki Tanaka; Fujita Health University (Toyoake): Tomoyuki Nakamura, Daisuke Niimi; Rakwakai Marutamachi Hospital (Kyoto): Takeshi Ueda; Osaka University Hospital (Suita City, Osaka Prefecture): Yozo Kashiwa, Akinori Uchiyama.

LATVIA: Paul Stradins Clinical University Hospital (Riga): Olegs Sabelnikovs, Peteris Oss.

LEBANON: Kortbawi Hospital (Jounieh): Youssef Haddad.

MALAYSIA: Hospital Kapit (Kapit): Kong Y. Liew.

MEXICO: Instituto Nacional De Cancerología, México (Mexico City): Silvio A. Namendys-Silva, Yves D. Jarquin-Badiola; Hospital De Especialidades "Antonio Fraga Mouret" Centro Medico Nacional La Raza IMSS (Mexico City): Luis A. Sanchez-Hurtado, Saira S. GomezFlores; Hospital Regional $1^{\circ}$ De Octubre (Mexico City): Maria C. Marin, Asisclo J. Villagomez; Hospital General Dr Manuel Gea Gonzalez (Mexico City): Jordana S. Lemus, Jonathan M. Fierro; Hospital General De Zona No. 1 Instituto Mexicano Del Seguro Social Tepic Nayarit (Tepic): Mavy Ramirez Cervantes, Francisco Javier Flores Mejia; Centro Medico Dalinde (Mexico D.F.): Dulce Dector, Dulce M. Dector; Opd Hospital Civil De Guadalajara Hospital Juan I Menchaca (Guadalajara): Daniel R. Gonzalez, Claudia R. Estrella; Hospital Regional De Ciudad 
Madero Pemex (Ciudad Madero): Jorge R. SanchezMedina, Alvaro Ramirez-Gutierrez; Centro Médico ABC (Mexico D.F.): Fernando G. George, Janet S. Aguirre; Hospital Juarez De Mexico (Mexico City): Juan A. Buensuseso, Manuel Poblano.

MOROCCO: Mohammed V University, University Teaching Ibn Sina Hospital (Rabat): Tarek Dendane, Amine Ali Zeggwagh; Hopital Militaire D'Instruction Mohammed V (Rabat): Hicham Balkhi; Errazi (Marrakech): Mina Elkhayari, Nacer Samkaoui; University Teaching Hospital Ibn Rushd (Casablanca): Hanane Ezzouine, Abdellatif Benslama; Hôpital des Spécialités de Rabat (HSR) (Rabat): Mourad Amor, Wajdi Maazouzi.

NETHERLANDS: Tjongerschans (Heerenveen): Nedim Cimic, Oliver Beck; Cwz (Nijmegen): Monique M. Bruns, Jeroen A. Schouten; Rijnstate Hospital (Arnhem): Myra Rinia, Monique Raaijmakers; Radboud Umc (Nijmegen): Leo M. Heunks, Hellen M. Van Wezel; Maastricht University Medical Centre (Maastricht): Serge J. Heines, Ulrich Strauch; Catharinaziekenhuis (Eindhoven): Marc P. Buise; Academic Medical Center (Amsterdam): Fabienne D. Simonis, Marcus J. Schultz.

NEW ZEALAND: Tauranga Hospital (Tauranga): Jennifer C. Goodson, Troy S. Browne; Wellington Hospital (Wellington): Leanlove Navarra, Anna Hunt; Dunedin Hospital (Dunedin): Robyn A. Hutchison, Mathew B. Bailey; Auckland City Hospital (Auckland): Lynette Newby, Colin Mcarthur; Whangarei Base Hospital (Whangarei): Michael Kalkoff, Alex Mcleod; North Shore Hospital (Auckland): Jonathan Casement, Danielle J. Hacking.

NORWAY: Ålesund Hospital (Ålesund): Finn $\mathrm{H}$. Andersen, Merete S. Dolva; Oslo University HospitalRikshospitalet Medical Centre (Oslo): Jon H. Laake, Andreas Barratt-Due; Stavanger University Hospital (Stavanger): Kim Andre L. Noremark, Eldar Søreide; Haukeland University Hospital (Bergen): Brit Å. Sjøbø, Anne B. Guttormsen.

PERU: Hospital Nacional Edgardo Rebagliati Martins (Lima): Hector H. Leon Yoshido; Clínica Ricardo Palma (Lima): Ronald Zumaran Aguilar, Fredy A. Montes Oscanoa.

PHILIPPINES: The Medical City (Pasig): Alain U. Alisasis, Joanne B. Robles; Chong Hua Hospital (Cebu): Rossini Abbie B. Pasanting-Lim, Beatriz C. Tan.

POLAND: Warsaw University Hospital (Warsaw): Pawel Andruszkiewicz, Karina Jakubowska.
PORTUGAL: Centro Hospitalar Da Cova Da Beira (Covilhã): Cristina M. Coxo; Hospital Santa Maria, Chln (Lisboa): António M. Alvarez, Bruno S. Oliveira; Centro Hospitalar Trás-Os-Montes E Alto Douro-Hospital De S.Pedro-Vila Real (Vila Real): Gustavo M. Montanha, Nelson C. Barros; Hospital Beatriz Ângelo (Loures): Carlos S. Pereira, António M. Messias; Hospital De Santa Maria (Lisboa): Jorge M. Monteiro; Centro Hospitalar Médio Tejo-Hospital De Abrantes (Abrantes): Ana M. Araujo, Nuno T. Catorze; Instituto Português De Oncologia De Lisboa (Lisboa): Susan M. Marum, Maria J. Bouw; Hospital Garcia De Orta (Almada): Rui M. Gomes, Vania A. Brito; Centro Hospitalar Do Algarve (Faro): Silvia Castro, Joana M. Estilita; HPP Hospital De Cascais (Alcabideche): Filipa M. Barros; Hospital Prof. Doutor Fernando Fonseca Epe (Amadora): Isabel M. Serra, Aurelia M. Martinho.

ROMANIA: Fundeni Clinical Institute (Bucharest): Dana R. Tomescu, Alexandra Marcu; Emergency Clinical County Hospital Timisoara (Timisoara): Ovidiu H. Bedreag, Marius Papurica; Elias University Emergency Hospital (Bucharest): Dan E. Corneci, Silvius Ioan Negoita.

RUSSIAN FEDERATION: University Hospital (Kemerovo): Evgeny Grigoriev; Krasnoyarsk Regional Hospital, Krasnoyarsk State Medical University (Krasnoyarsk): Alexey I. Gritsan, Andrey A. Gazenkampf.

SAUDI ARABIA: GICU of PSMMC (Riyadh): Ghaleb Almekhlafi, Mohamad M. Albarrak; SICU of PSMMC (Riyadh): Ghanem M. Mustafa; King Faisal Hospital and Research Center (Riyadh): Khalid A. Maghrabi, Nawal Salahuddin; King Fahad Hospital (Baha): Tharwat M. Aisa; King Abdulaziz Medical City (Riyadh): Ahmed S. Al Jabbary, Edgardo Tabhan; King Abdulaziz Medical City (Riyadh): Yaseen M. Arabi; King Abdulaziz Medical City (Riyadh): Yaseen M. Arabi, Olivia A. Trinidad; King Abdulaziz Medical City (Riyadh): Hasan M. Al Dorzi, Edgardo E. Tabhan.

SOUTH AFRICA: Charlotte Maxeke Johannesburg Academic Hospital (Johannesburg): Stefan Bolon, Oliver Smith.

SPAIN: Hospital Sant Pau (Barcelona): Jordi Mancebo, Hernan Aguirre-Bermeo; Hospital Universitari Bellvitge (L Hospitalet De Llobregat (Barcelona)): Juan C. LopezDelgado, Francisco Esteve; Hospital Son Llatzer (Palma De Mallorca): Gemma Rialp, Catalina Forteza; Sabadell Hospital, CIBER Enfermedades Respiratorias (Sabadell): Candelaria De Haro, Antonio Artigas; Hospital 
Universitario Central De Asturias (Oviedo): Guillermo M. Albaiceta, Sara De Cima-Iglesias; Complejo Hospitalario Universitario A Coruña (A Coruña): Leticia Seoane-Quiroga, Alexandra Ceniceros-Barros; Hospital Universitario Miguel Servet (Zaragoza): Antonio L. RuizAguilar, Luis M. Claraco-Vega; Morales Meseguer University Hospital (Murcia): Juan Alfonso Soler, Maria del Carmen Lorente; Hospital Universitario del Henares (Coslada): Cecilia Hermosa, Federico Gordo; Complejo Asistencial De Palencia. Hospital Rio Carrión (Palencia): Miryam Prieto-González, Juan B. López-Messa; Fundación Jiménez Díaz (Madrid): Manuel P. Perez, Cesar P. Perez; Hospital Clínico Universitario Lozano Blesa (Zaragoza): Raquel Montoiro Allue; Hospital Verge de la Cinta (Tortosa): Ferran Roche-Campo, Marcos Ibañez-Santacruz; Hospital Universitario 12 De Octubre (Madrid): Susana Temprano; Hospital Universitario Príncipe De Asturias (Alcalá De Henares, Madrid): Maria C. Pintado, Raul De Pablo; Hospital Universitari Germans Trias I Pujol (Badalona): Pilar Ricart Aroa Gómez; Hospital Universitario Arnau De Vilanova De Lleida (Lleida): Silvia Rodriguez Ruiz, Silvia Iglesias Moles; Cst Terrassa (Barcelona): $\mathrm{M}^{\mathrm{a}}$ Teresa Jurado, Alfons Arizmendi; Hospital Universitari Mútua Terrassa (Terrassa): Enrique A. Piacentini; Hospital Universitario De Móstoles (Mostoles): Nieves Franco, Teresa Honrubia; Complejo Asistencial De Salamanca (Salamanca): Meisy Perez Cheng, Elena Perez Losada; Hospital General Universitario De Ciudad Real (Ciudad Real): Javier Blanco, Luis J. Yuste; Torrecardenas (Almeria): Cecilia Carbayo-Gorriz, Francisca G. Cazorla-Barranquero; Hospital Universitario Donostia (San Sebastian): Javier G. Alonso, Rosa S. Alda; Hospital Universitario De Torrejón (Madrid): Ángela Algaba, Gonzalo Navarro; Hospital Universitario De La Princesa (Madrid): Enrique Cereijo, Esther DiazRodriguez; Hospital Universitario Lucus Augusti (Lugo): Diego Pastor Marcos, Laura Alvarez Montero; Hospital Universitario Santa Lucia (Cartagena): Luis Herrera Para, Roberto Jimenez Sanchez; Hospital Universitario Severo Ochoa, Leganes (Madrid): Miguel Angel Blasco Navalpotro, Ricardo Diaz Abad; University Hospital of Ntra. Sra. De Candelaria (Santa Cruz De Tenerife): Raquel Montiel González, Dácil Parrilla Toribio; Hospital Universitario Marques De Valdecilla (Santander): Alejandro G. Castro, Maria Jose D. Artiga; Hospital Infanta Cristina (Parla, Madrid): Oscar Penuelas; Hospital General De Catalunya (Sant Cugat Del Valles): Tomas P. Roser, Moreno F. Olga; San Pedro De Alcántara (Cáceres): Elena Gallego Curto, Rocío Manzano Sánchez; Sant Joan De Reus (Reus): Vallverdu P. Imma, Garcia M. Elisabet; Hospital Joan XXIII (Tarragona): Laura Claverias, Monica Magret; Hospital Universitario De Getafe (Madrid):
Ana M. Pellicer, Lucia L. Rodriguez; Hospital Universitario Río Hortega (Valladolid): Jesús Sánchez-Ballesteros, Ángela González-Salamanca; Hospital Arquitecto Marcide (Ferrol, La Coruña): Antonio G. Jimenez, Francisco P. Huerta; Hospital General Universitario Gregorio Marañón (Madrid): Juan Carlos J. Sotillo Diaz, Esther Bermejo Lopez; Hospital General De Segovia (Segovia): David D. Llinares Moya, Alec A. Tallet Alfonso; Hospital General Universitario Reina Sofia (Murcia): Palazon Sanchez Eugenio Luis, Palazon Sanchez Cesar; Complejo Hospitalario Universitario De Albacete (Albacete): Sánchez I. Rafael, Corcoles G. Virgilio; Hospital Infanta Elena (Valdemoro): Noelia N. Recio.

SWEDEN: Sahlgrenska University Hospital (Gothenburg): Richard O. Adamsson, Christian C. Rylander; Karolinska University Hospital (Stockholm): Bernhard Holzgraefe, Lars M. Broman; Akademiska Sjukhuset Uppsala (Uppsala): Joanna Wessbergh, Linnea Persson; Vrinnevisjukhuset (Norrköping): Fredrik Schiöler, Hans Kedelv; Linkoping University Hospital (Linköping): Anna Oscarsson Tibblin, Henrik Appelberg; Skellefteå Lasarett (Skellefteå): Lars Hedlund, Johan Helleberg; Karolinska University Hospital Solna (Stockholm): Karin E. Eriksson, Rita Glietsch; Umeå University Hospital (Umeå): Niklas Larsson, Ingela Nygren; Danderyd Hospital (Stockholm): Silvia L. Nunes, Anna-Karin Morin; Lund University Hospital (Lund): Thomas Kander, Anne Adolfsson.

SWITZERLAND: Chuv (Centre Hospitalier Universitaire Vaudois) (Lausanne): Lise Piquilloud; Hôpital neuchâtelois-La Chaux-De-Fonds (La Chaux-De-Fonds): Hervé O. Zender, Corinne Leemann-Refondini.

TUNISIA: Hopital Tahar Sfar Mahdia (Mahdia): Souheil Elatrous; University Hospital Farhat Hached Sousse (Sousse): Slaheddine Bouchoucha, Imed Chouchene; CHU F.Bourguiba (Monastir): Islem Ouanes; Mongi Slim University Hospital, La Marsa (La Marsa): Asma Ben Souissi, Salma Kamoun.

TURKEY: Cerrahpasa Medical Faculty Emergency Intensive Care Unit (Istanbul): Oktay Demirkiran; Cerrahpasa Medical Faculty Sadi Sun Intensive Care Unit (Istanbul): Mustafa Aker, Emre Erbabacan; Uludag University Medical Faculty (Bursa): Ilkay Ceylan, Nermin Kelebek Girgin; Ankara University Faculty of Medicine, Reanimation 3nd level ICU (Ankara): Menekse Ozcelik, Necmettin Ünal; Ankara University Faculty of Medicine, 2nd level ICUpostoperative ICU (Ankara): Basak Ceyda Meco; Istanbul Kartal Egitim Ve Arastirma Hastanesi (Istanbul): Onat O. Akyol, Suleyman S. Derman. 
UNITED KINGDOM: Papworth Hospital (Cambridge): Barry Kennedy, Ken Parhar; Royal Glamorgan Hospital (Llantrisant): Latha Srinivasa; Royal Victoria HospitalBelfast (Belfast): Lia McNamee, Danny McAuley; Jack Steinberg ICU of the King's College (London): Phil Hopkins, Clare Mellis; Frank Stansil ICU of the King's College Hospital (London): Vivek Kakar; Liver ICU of the King's College (London): Dan Hadfield; Christine Brown ICU of the King's College (London): Andre Vercueil; West Suffolk Hospital (Bury St Edmunds): Kaushik Bhowmick, Sally K. Humphreys; Craigavon Area Hospital (Portadown): Andrew Ferguson, Raymond Mckee; Barts Health NHS Trust, Whipps Cross Hospital (Leytonstone): Ashok S. Raj, Danielle A. Fawkes; Kettering General Hospital, Foundation NHS Trust (Northamptonshire): Philip Watt, Linda Twohey; Barnet General Hospital (Barnet): Rajeev R. JhaMatthew Thomas, Alex Morton, Varsha Kadaba; Rotherham General Hospital (Rotherham): Mark J. Smith, Anil P. Hormis; City Hospital (Birmingham): Santhana G. Kannan, Miriam Namih; Poole Hospital NHS Foundation Trust (Poole): Henrik Reschreiter, Julie Camsooksai; Weston General Hospital (Weston-Super-Mare): Alek Kumar, Szabolcs Rugonfalvi; Antrim Area Hospital (Antrim): Christopher Nutt, Orla Oneill; Aintree University Hospital (Liverpool): Colette Seasman, Ged Dempsey; Northern General Hospital (Sheffield): Christopher J. Scott, Helen E. Ellis; John Radcliffe Hospital (Oxford): Stuart Mckechnie, Paula J. Hutton; St Georges Hospital (London): Nora N. Di Tomasso, Michela N. Vitale; Hillingdon Hospital (Uxbridge): Ruth O. Griffin, Michael N. Dean; The Royal Bournemouth and Christchurch NHS Foundation Trust (Bournemouth, Dorset): Julius $\mathrm{H}$. Cranshaw, Emma L. Willett; Guys and St Thomas NHS Foundation Trust (London): Nicholas Ioannou, Gstt Severe Respiratory Failure Service; Whittington Hospital (London): Sarah Gillis; Wexham Park Hospital (Slough): Peter Csabi; Western General Hospital (Edinburgh): Rosaleen Macfadyen, Heidi Dawson; Royal Preston Hospital (Preston): Pieter D. Preez, Alexandra J. Williams; Brighton and Sussex University Hospitals NHS Trust (Brighton): Owen Boyd, Laura Ortiz-Ruiz De Gordoa; East and North Herts NHS Trust (Stevenage): Jon Bramall, Sophie Symmonds; Barnsley Hospital (Barnsley): Simon K. Chau, Tim Wenham; Prince Charles Hospital (Merthyr Tydfil): Tamas Szakmany, Piroska Toth-Tarsoly; University Hospital of South Manchester NHS Foundation Trust (Manchester): Katie H. Mccalman, Peter Alexander; Harrogate District Hospital (Harrogate): Lorraine Stephenson, Thomas Collyer; East and North Herts NHS Trust (Welwyn Garden City): Rhiannon Chapman, Raphael Cooper; Western Infirmary (Glasgow): Russell M. Allan, Malcolm Sim; Dumfries and Galloway Royal Infirmary (Dumfries): David W. Wrathall, Donald A. Irvine;
Charing Cross Hospital (London): Kim S. Zantua, John C. Adams; Worcestershire Royal Hospital (Worcester): Andrew J. Burtenshaw, Gareth P. Sellors; Royal Liverpool University Hospital (Liverpool): Ingeborg D. Welters, Karen E. Williams; Royal Alexandra Hospital (Glasgow): Robert J. Hessell, Matthew G. Oldroyd; Morriston Hospital (Swansea): Ceri E. Battle, Suresh Pillai; Frimley Park Hospital (Frimley): Istvan Kajtor, Mageswaran Sivashanmugavel; Altnagelvin Hospital (Derry): Sinead C. Okane, Adrian Donnelly; Buckinghamshire Healthcare NHS Trust (High Wycombe, Buckinghamshire): Aniko D. Frigyik, Jon P. Careless; Milton Keynes Hospital (Milton Keynes): Martin M. May, Richard Stewart; Ulster Hospital (Belfast): T. John Trinder, Samantha J. Hagan; University Hospital of Wales (Cardiff): Matt P. Wise, Jade M. Cole; Freeman Hospital (Newcastle Upon Tyne): Caroline C. MacFie, Anna T. Dowling.

URUGUAY: Hospital Español (Montevideo): Javier Hurtado, Nicolás Nin; Cudam (Montevideo): Javier Hurtado; Sanatorio Mautone (Maldonado): Edgardo Nuñez; Sanatorio Americano (Montevideo): Gustavo Pittini, Ruben Rodriguez; Hospital De Clínicas (Montevideo): María C. Imperio, Cristina Santos; Circulo Católico Obreros Uruguay-Sanatorio JPII (Montevideo): Ana G. França, Alejandro Ebeid; CASMU (Montevideo): Alberto Deicas, Carolina Serra.

USA: Saint Louis University Hospital (St. Louis): Aditya Uppalapati, Ghassan Kamel; Beth Israel Deaconess Medical Center (Boston): Valerie M. Banner-Goodspeed, Jeremy R. Beitler; Memorial Medical Center (Springfield): Satyanarayana Reddy Mukkera, Shreedhar Kulkarni; Massachusetts General Hospital (Boston): Jarone Lee, Tomaz Mesar; University of Cincinnati Medical Center (Cincinnati): John O. Shinn Iii, Dina Gomaa; Massachusetts General Hospital (Boston): Christopher Tainter, Jarone Lee; Massachusetts General Hospital (Boston): Tomaz Mesar, Jarone Lee; R Adams Cowley Shock Trauma Center (Baltimore): Dale J. Yeatts, Jessica Warren; Intermountain Medical Center (Murray, Utah): Michael J. Lanspa, Russel R. Miller; Intermountain Medical Center (Murray, Utah): Colin K. Grissom, Samuel M. Brown; Mayo Clinic (Rochester): Philippe R. Bauer; North Shore Medical Center (Salem): Ryan J. Gosselin, Barrett T. Kitch; Albany Medical Center (Albany): Jason E. Cohen, Scott H. Beegle; John H. Stoger Hospital of Cook County (Chicago, Il): Renaud M. Gueret, Aiman Tulaimat; Albany Medical Center (Albany): Shazia Choudry; University of Alabama at Birmingham (UAb) (Birmingham, AL): William Stigler, Hitesh Batra; Duke University Hospital (Durham): Nidhi G. Huff; Iowa Methodist Medical Center (Des Moines, Iowa): Keith D. Lamb, 
Trevor W. Oetting; Surgical and Neurosciences Intensive Care Unit of the University of Iowa Hospitals and Clinics (Iowa City, Iowa): Nicholas M. Mohr, Claine Judy; Medical Center of Louisiana at New Orleans (New Orleans, Louisiana): Shigeki Saito, Fayez M. Kheir; Tulane University (New Orleans): Fayez Kheir; Critical Care Unit of the University of Iowa Hospitals and Clinics (Iowa City, Iowa): Adam B. Schlichting, Angela Delsing; University of California, San Diego Medical Center (San Diego, CA): Daniel R. Crouch, Mary Elmasri; UC San Diego Thornton Hospital (La Jolla): Daniel R. Crouch, Dina Ismail; University Hospital (Cincinnati): Kyle R. Dreyer, Thomas C. Blakeman; University Hospital (Cincinnati): Kyle R. Dreyer, Dina Gomaa; Tower 3B Medical ICU of Brigham and Women's Hospital (Boston): Rebecca M. Baron, Carolina Quintana Grijalba; Tower 8C Burn/Trauma ICU of Brigham and Women's Hospital (Boston): Peter C. Hou; Tower 8D Surgical ICU of Brigham and Women's Hospital (Boston): Raghu Seethala; Tower 9C Neurosurgical ICU of Brigham and Women's Hospital (Boston): Imo Aisiku; Tower 9D Neurological ICU of Brigham and Women's Hospital (Boston): Galen Henderson; Tower $11 \mathrm{C}$ Thoracic ICU of Brigham and Women's Hospital (Boston): Gyorgy Frendl; Shapiro 6W Cardiac Surgery ICU of Brigham and Women's Hospital (Boston): SenKuang Hou; Shapiro 9E Coronary Care Unit of Brigham and Women's Hospital (Boston): Robert L. Owens, Ashley Schomer.

SERBIA: Clinical Center of Serbia (Belgrade): Vesna Bumbasirevic, Bojan Jovanovic; Military Medical Academy (Belgrade): Maja Surbatovic, Milic Veljovic.

\section{LUNG SAFE Study Team}

\author{
Steering Committee \\ Laurent Brochard \\ Andres Esteban \\ Luciano Gattinoni \\ Frank van Haren \\ John Laffey \\ Anders Larsson \\ Danny McAuley \\ Antonio Pesenti (CHAIR) \\ Marco Ranieri \\ Gordon Rubenfeld \\ Art Slutsky \\ B. Taylor Thompson \\ Hermann Wrigge
}

\author{
Executive Committee \\ Giacomo Bellani \\ Eddy Fan \\ John Laffey (CHAIR) \\ Tai Pham
}

\section{Author details}

1 Departments of Anesthesia and Critical Care Medicine, Keenan Research Centre for Biomedical Science, St Michael's Hospital, Toronto, Canada.

${ }^{2}$ Departments of Anesthesia, Physiology, Interdepartmental Division of Critical Care Medicine, University of Toronto, Toronto, Canada. ${ }^{3}$ School of Medicine and Surgery, University of Milan-Bicocca, Monza, Italy. ${ }^{4}$ Department of Emergency and Intensive Care, San Gerardo Hospital, Monza, Italy.

${ }^{5}$ AP-HP, Hôpital Tenon, Unité de Réanimation médico-chirurgicale, Pôle Thorax Voies aériennes, Groupe hospitalier des Hôpitaux Universitaires de l'Est Parisien, Paris, France. ${ }^{6}$ UMR 1153, Inserm, Sorbonne Paris Cité, ECSTRA Team, Université Paris Diderot, Paris, France. ${ }^{7}$ UMR 915, Inserm, Université Paris Est Créteil, Créteil, France. ${ }^{8}$ Department of Medicine, University Health Network, Mount Sinai Hospital, Toronto, Canada. ${ }^{9}$ Interdepartmental Division of Critical Care Medicine and Institute of Health Policy, Management and Evaluation, University of Toronto, Toronto, Canada. ${ }^{10}$ Research Center on Public Health, School of Medicine and Surgery, University of Milano-Bicocca, Monza, Italy. ${ }^{11}$ Division of Pulmonary and Critical Care Unit, Department of Medicine, Massachusetts General Hospital, Harvard Medical School, Boston, MA, USA.

12 Keenan Research Centre, Li Ka Shing Knowledge Institute, St Michael's Hospital, Toronto, Canada. ${ }^{13}$ Interdepartmental Division of Critical Care Medicine, University of Toronto, Toronto, Canada. ${ }^{14}$ Department of Anaesthesia, National University of Ireland, Galway University Hospitals, Galway, Ireland. ${ }^{15}$ Hospital Universitario de Getafe, CIBER de Enfermedades Respiratorias, Madrid, Spain.

${ }^{16}$ Department of Anesthesiology, Emergency and Intensive Care Medicine Universitätsmedizin Göttingen, Robert Koch Strasse 40, 37073 Göttingen, Germany. ${ }^{17}$ Intensive Care Unit, The Canberra Hospital, Australian National University, Canberra, Australia. ${ }^{18}$ Department of Intensive Care Medicine, Radboud University Medical Center, Nijmegen, The Netherlands. ${ }^{19}$ Department of Anesthesiology, Yokohama City University Medical Center, Kanagawa, Japan.

${ }^{20}$ Division of Critical Care, Department of Anaesthesiology, Rikshospitalet Medical Centre, Oslo University Hospital, Oslo, Norway. ${ }^{21}$ Section of Anesthesiology and Intensive Care, Department of Surgical Sciences, Uppsala University, Uppsala, Sweden. ${ }^{22}$ Centre for Experimental Medicine, Queen's University of Belfast, Wellcome-Wolfson Institute for Experimental Medicine, Belfast and Regional Intensive Care Unit, Royal Victoria Hospital, Grosvenor Road, Belfast, Northern Ireland, UK. ${ }^{23}$ Department of Critical Care Medicine, Nanjing Zhongda Hospital, School of Medicine, Southeast University, Nanjing 210009, People's Republic of China. ${ }^{24}$ SAPIENZA Università di ROMA, Dipartimento di Anestesia e Rianimazione, Policlinico Umberto I, Viale del Policlinico 155, 00161 Rome, Italy. ${ }^{25}$ Interdepartmental Division of Critical Care Medicine, University of Toronto and Program in Trauma, Emergency and Critical Care, Sunnybrook Health Sciences Center, Toronto, Canada. ${ }^{26}$ Department of Anesthesiology and Intensive Care Medicine, University of Leipzig, 04103 Leipzig, Germany. ${ }^{27}$ Department of Medicine, University of Toronto, Toronto, Canada. ${ }^{28}$ Dipartimento di Anestesia, Rianimazione ed Emergenza Urgenza, Fondazione IRCCS Cà Granda-Ospedale Maggiore Policlinico, Toronto, Canada. ${ }^{29}$ Dipartimento di Fisiopatologia Medico-Chirurgica e dei Trapianti, Università degli Studi di Milano, Milan, Italy.

Published online: 14 November 2017 